\title{
Recomendações nutricionais para o paciente com câncer durante a pandemia do coronavírus (COVID-19)
}

Nutritional recommendations for cancer patients during the coronavirus pandemic (COVID-19)

\section{DOI: 10.37111/braspenj.AE2020351002}

Juliana Moura Nabarrete'

Cristiane A. D'Almeida

Melina Gouveia Castro

Diogo Oliveira Toledo ${ }^{4}$

\section{Unitermos:}

Recomendações nutricionais. Neoplasias. Coronavírus. COVID-19. SARS-CoV-2. Pandemias.

\section{Keywords:}

Recommended dietary allowances. Neoplasms. Coronavirus. COVID-19. SARS-CoV-2, Pandemics.

\section{Endereço para correspondência:}

Juliana Moura Nabarrete

Rua Abílio Soares, 233 - cj 144 - Paraíso - São Paulo, SP, Brasil - CEP: 04005-000

E-mail: juliana.m.nabarrete@gmail.com

\section{Submissão}

12 de março de 2020

Aceito para publicação

25 de março de 2020

\section{RESUMO}

Pacientes com câncer em tratamento encontram-se no grupo de risco para COVID-19. Estes pacientes possuem predisposição a maior gravidade da infecção ocasionada pelo coronavírus. Durante o cenário atual do quadro desta pandemia, a nutrição foi citada inúmeras vezes pelo fato de contribuir com o tratamento no geral. Infelizmente, os relatos da assistência nutricional ao paciente oncológico neste momento ainda são escassos. $\mathrm{O}$ objetivo deste documento é discutir as condutas recomendadas em um compilado dos dados já publicados sobre nutrição e coronavírus, neste momento específico.

\section{ABSTRACT}

Cancer patients undergoing treatment are at risk for COVID-19. These patients are predisposed to greater severity of infection caused by the coronavirus. During the current scenario of this pandemic, nutrition has been cited numerous times by the fact contribute to the treatment in general. Unfortunately, reports of nutritional care for cancer patients are currently scarce. The purpose of this document is to discuss the recommended practices in a compilation of data already published on nutrition and coronavirus, at this specific time.

1. Especialista em Nutrição Clínica em Pediatria do Instituto da Criança do Hospital das Clínicas da Faculdade de Medicina da Universidade de São Paulo, Nutricionista do Centro Integrado de Oncologia - Oncologistas Associados e do Radium Instituto de Oncologia, São Paulo, SP, Brasil.

2. Doutora em Ciências Nutricionais pela Universidade Federal do Rio de Janeiro (UFRJ), Nutricionista do Instituto Nacional de Câncer José Alencar Gomes da Silva, Rio de Janeiro, RJ, Brasil.

3. Doutora em Cirurgia do Aparelho Digestivo pela Faculdade de Medicina da Universidade de São Paulo, Especialista em Terapia Nutricional Enteral e Parenteral pela BRASPEN, Médica Nutróloga do Departamento de Terapia Nutricional do Hospital Israelita Albert Einstein, São Paulo, SP, Brasil.

4. Mestre em Ciências pelo Instituto de Assistência Médica ao Servidor Público Estadual de São Paulo (IAMSPE), Especialista em Terapia Nutricional Enteral e Parenteral pela BRASPEN, Coordenador do Departamento de Terapia Nutricional do Hospital Israelita Albert Einstein, São Paulo, SP, Brasil. 


\section{INTRODUÇÃO}

Os pacientes com câncer se posicionam no grupo de risco para COVID-19 por apresentarem uma diminuição da imunidade. Isso se deve à própria doença e/ou pelo resultado imunossupressor dos tratamentos propostos, bem como a situação pós-cirúrgica, quimioterapia, uso de corticosteroides, transfusões sanguíneas, radioterapia e transplantes.

Com a declaração da Organização Mundial de Saúde de pandemia, em 11 de março de 2020, houve crescente número de informações sobre a doença, seus efeitos, possíveis formas de tratamento e de prevenção. A nutrição, por sua vez, foi citada inúmeras vezes como parte do manejo.

O Conselho Federal de Nutrição emitiu uma nota oficial com recomendações, informando que "não existem protocolos técnicos nem evidências científicas que sustentem alegações milagrosas". É importante reforçar que a melhor conduta para a população, neste momento, seria a adoção rigorosa de medidas preventivas e protetivas ao coronavírus indicadas pelo Ministério da Saúde. Notas divulgadas sem cunho científico envolvendo alimentos e terapias nutricionais com a cura da doença são informações que devem ser abolidas. Desta forma, as melhores práticas são buscar: medidas como acessar informações sobre alimentação de fontes confiáveis, conhecer o Guia Alimentar para a População Brasileira e procurar um profissional nutricionista para orientação nutricional.

O profissional nutricionista deve reforçar a necessidade de uma análise crítica das questões técnico-científicas e metodológicas de práticas. Além disso, atualizar-se constantemente sobre protocolos divulgados com respaldo técnico-científico, compartilhar informações visando à promoção da saúde e à educação alimentar. Já aos que se mantiverem atuando profissionalmente, que atendam às orientações das autoridades sanitárias quanto à proteção individual e possibilidades de quarentena.

Especificamente na Oncologia, até o presente momento, os tratamentos oncológicos não estão suspensos. Esta é uma decisão da equipe médica a ser avaliada individualmente. Algumas internações ocorrerão, independente do COVID19, por possíveis complicações do paciente oncológico durante o tratamento. A imensa maioria manterá acompanhamento ambulatorial, exigindo um novo fluxo de assepsia do ambiente e agendamento de toda equipe interdisciplinar, visando evitar aglomerações.

Os relatos da assistência nutricional ao paciente com câncer, neste momento, ainda são escassos. A abordagem focará em segurança alimentar, terapia nutricional via oral, terapia nutricional enteral, assistência nutricional ao paciente internado e assistência nutricional ambulatorial.

\section{SEGURANÇA ALIMENTAR}

Um compilado de artigos demonstrou a eficácia de adotar medidas de segurança alimentar, tais como: cuidados na higienização e armazenamento dos alimentos, como frutas, verduras e legumes crus, fontes ricas em micronutrientes e compostos bioativos, tão importantes neste momento para o fortalecimento do sistema imunológico.

Sabemos que as condutas de higiene são medidas eficazes no combate ao coronavírus. As orientações de segurança alimentar para o paciente em acompanhamento ambulatorial podem ser respaldadas por documentos publicados pelo Food Drug Administration (FDA) ou pela Agência Nacional de Vigilância Sanitária (ANVISA). A mais utilizada é Resolução RDC 12/2001, que estabelece os Padrões Microbiológicos Sanitários para Alimentos e determina os critérios para a conclusão e interpretação dos resultados das análises microbiológicas de alimentos destinados ao consumo humano.

Desta forma, é importante reforçar as orientações de segurança alimentar usuais, como cuidados na aquisição, preparo e armazenamento destes alimentos para todos os pacientes oncológicos, diagnosticados ou não com COVID-19:

- Priorizar o consumo de refeições preparadas em casa;

- Planejar as refeições que farão parte do cardápio semanal/quinzenal da residência, evitando, assim, idas frequentes ao mercado dos familiares próximos ao paciente;

- Ao chegar do mercado, higienizar as embalagens de plástico, papel, metal ou vidro com papel toalha ou um pano limpo com álcool $70^{\circ}$. Caso o paciente opte por um pano, deverá realizar a lavagem imediata antes de guardar;

- Realizar lavagem das mãos em água e sabão antes de qualquer contato com os alimentos, seja para preparo ou consumo;

- Orientar a compra de frutas e vegetais que estejam no momento de safra, facilitando a aquisição de produtos com as características usuais do alimento e, sempre que possível, de produção orgânica;

- Realizar a higienização de vegetais e frutas com água clorada (cloro orgânico ou hipoclorito de sódio), utilizando a concentração entre 100 a 200 ppm por 15 minutos ou conforme instruções da embalagem de cada produto;

- Evitar estocagem de grandes quantidades de alimentos. Se não houver um controle da quantidade de comida na residência, pode ocorrer descontrole com a data de validade dos alimentos e criar um ambiente propício à disseminação de patógenos alimentares; 
- Reforçar o consumo de carnes em geral, preparadas em métodos de cocção, no qual o resultado seja uma cor uniforme, identificando que o alimento está totalmente cozido, independentemente do método adotado (grelhar, cozinhar, assar);

- Reforçar os cuidados de higiene no preparo das refeições: lavagem de mãos, manter as unhas curtas, limpeza frequente do ambiente em que se realiza o preparo, bem como coleta constante do lixo;

- Não compartilhar talheres, copos, pratos ou garrafas no momento das refeições. Realizar a lavagem dos utensílios ao término da refeição com água e sabão.

Os estabelecimentos de internação hospitalar devem seguir as orientações da Resolução RDC n 216 , de 15 de setembro de 2004. Além disso, possuir um Manual de Boas Práticas de Fabricação de produtos para refeição. Para 0 momento da atual pandemia do COVID-19, a ANVISA publicou uma nota técnica de 04/2020 com orientações para os serviços de saúde quanto às medidas de prevenção e controle. Neste documento, a ANVISA reforça que todos os pacientes e acompanhantes devem ser orientados a não compartilhar objetos (pratos, copos, talheres) e alimentos com outros pacientes e acompanhantes ou entre si. Também são recomendadas medidas de prevenção e controle no que diz respeito à higienização e paramentação dos profissionais de saúde e de apoio (profissionais da higiene e limpeza, nutrição, manutenção, etc) ao contato com casos suspeitos ou confirmados e acompanhantes. Seguir as regras já determinadas, como higiene das mãos frequente com água e sabonete líquido ou preparação alcoólica a 70\%, gorro (para procedimentos que geram aerossóis), óculos de proteção ou protetor facial, máscara cirúrgica, avental e luvas de procedimentos.

Vale ressaltar que cada instituição deve estar sincronizada com todas as equipes, para alinhamento de rotina de utilização de equipamentos de proteção individual (EPI's). Além disso, faz-se necessário confirmar qual orientação da sua instituição com seu gestor, para adotar a conduta mais customizada para o estabelecimento.

\section{TERAPIA NUTRICIONAL VIA ORAL}

Por se tratar de uma população com a ingestão alimentar prejudicada devido à doença de base, devemos manter uma vigilância constante quanto à quantidade ingerida.

Os parâmetros utilizados para cálculo de necessidades calóricas e proteicas devem seguir inicialmente as últimas diretrizes já publicadas, conforme Quadros 1 e 2. Não havendo qualquer recomendação específica para pacientes com COVID-19, até o momento.

Para pacientes oncológicos com COVID-19 positivo, em bom estado geral e sem terapia nutricional oral atual, deve-se
Quadro 1-Recomendações de oferta energética para paciente com câncer.

Paciente com câncer adulto e idoso, em tratamento: 25 a $30 \mathrm{kcal} / \mathrm{kg} / \mathrm{dia}$

Paciente com câncer idoso com IMC < 18,5 kg/m²: 32 a 38 kcal $/ \mathrm{kg} / \mathrm{dia}$

Pacientes com câncer e obesidade: 20 a $25 \mathrm{kcal} / \mathrm{kg} / \mathrm{dia}$

Pacientes com câncer e caquexia ou desnutridos: 30 a 35 kcal $/ \mathrm{kg} / \mathrm{dia}$

Quadro 2 - Recomendações de oferta proteica para paciente com câncer.

Paciente com câncer adulto e idoso em tratamento antineoplásico: acima de $1,0 \mathrm{~g}$ e se a inflamação sistêmica for presente considerar 1,2 a 2,0 $\mathrm{g} / \mathrm{kg} / \mathrm{dia}$

Pacientes com algum grau de desnutrição: 1,2 a 1,5 g/kg/dia

manter acompanhamento da aceitação alimentar frequente, por meio de recordatório alimentar habitual ou $24 \mathrm{~h}$ e iniciar suplementação via oral precoce, a fim de evitar agravo do estado nutricional.

Para pacientes oncológicos sem o diagnóstico de COVID19, é recomendado:

- Manter a suplementação via oral usualmente utilizada, reforçando sua importância;

- Fortalecer a alimentação via oral diversificada, baseada em refeições que contenham todos os grupos alimentares e associem micronutrientes (minerais e vitaminas) e substâncias bioativas (não nutrientes), respeitando os efeitos colaterais do tratamento;

- Não indicar "superalimentos", shots, sucos ou soroterapias por infusão endovenosa de nutrientes (vitaminas, minerais, aminoácidos, antioxidantes e outros nutrientes e compostos) como formas de prevenção ou combate ao coronavírus.

\section{TERAPIA NUTRICIONAL VIA ENTERAL}

Pacientes oncológicos em uso de terapia nutricional via enteral devem ter reforçadas as orientações de cuidados de higiene com o manuseio da dieta, equipo e sonda.

Quando necessário o suporte intensivo devido à associação do COVID-19 ao diagnóstico oncológico, deve-se avaliar a necessidade de utilizar referências específicas para este momento, conforme parecer recém-publicado pela BRASPEN. O paciente estável tem indicação de início precoce de nutrição enteral (24 a 48h), minimizando risco de desnutrição.

\section{ASSISTENACIA NUTRICIONAL AO PACIENTE INTERNADO}

Os tratamentos oncológicos não estão suspensos. Esta é uma decisão da equipe médica, a ser avaliada individualmente. Algumas internações ocorrerão, independente do COVID-19, por possíveis complicações do paciente oncológico durante o tratamento. 
- Seguir as recomendações de cada instituição sobre utilização de EPI's e higienização de equipamentos para avaliação nutricional;

- Priorizar a aplicação de ferramentas subjetivas de triagem e avaliação nutricional que atendam à necessidade do seu serviço neste momento: NRS-2002, MUST, ASG-PPP, MUST, MAN-VR.

\section{ASSISTÊNCIA NUTRICIONAL AO PACIENTE AMBULATORIAL}

Os tratamentos oncológicos de acompanhamento ambulatorial também não estão suspensos. Esta é uma decisão da equipe médica a ser avaliada individualmente, porém o fluxo de pacientes no ambulatório geral deve ser revisto e na nutrição também:

- Seguir as recomendações de cada instituição sobre utilização de EPI's e higienização de equipamentos para avaliação nutricional;

- Avaliar, criteriosamente, a manutenção do acompanhamento presencial, bem como métodos de avaliação antropométrica e composição corporal, devido a se tratar de uma população de risco devido à imunossupressão apresentada;

- O Conselho Federal de Nutricionistas publicou, em 18/03/2020, a Resolução CFN Nº 646, de 18 de março de 2020, que autoriza, em caráter de exceção, a assistência nutricional por meio não presencial até a data de 31/08/2020, respaldando a continuidade do acompanhamento dos pacientes com câncer, por exemplo;

- Em casos de assistência não presencial, priorizar a aplicação de ferramentas subjetivas de triagem e avaliação nutricional que atendam à necessidade do serviço neste momento: NRS-2002, MUST, ASG-PPP, MUST, MAN-VR;

- Realizar a avaliação da aceitação alimentar e sintomas.

\section{CONCLUSÃO}

O acompanhamento e intervenção nutricional são fundamentais para manutenção do estado nutricional, menor risco de complicações, melhora da qualidade de vida, melhor resposta ao tratamento oncológico. E, embora ainda existam poucas referências e direções a serem tomadas frente ao paciente com câncer, os cuidados nutricionais, de higiene e segurança alimentar, nesta população, devem ser encorajados e mantidos.

\section{REFERENCIAS}

1. Conselho Federal de Nutricionistas. Resolução CFN N ${ }^{\circ}$ 646, de 18 de março de 2020. [citado 2020 Mar 10]. Disponível em: https://www.cfn.org.br/wp-content/uploads/2020/03/ResolCFN-646-codigo-etica.pdf

2. Ueda M, Martins R, Hendrie PC, McDonnell T, Crews JR, Wong TJ, et al. Managing cancer care during the COVID-19 pandemic: agility and collaboration toward a common goal. J Natl Compr Canc Netw. 2020;20:1-4.

3. Harbige L, Calder P, Marcos A, Dardenne M, Perdigón G, PerezCano F, et al. ISIN Position Statement on Nutrition, Immunity and COVID-1. March 2020. [citado 2020 Mar 10]. Disponível em: http://www.immunonutrition-isin.org/

4. Zhang L, Liu Y. Potential interventions for novel coronavirus in China: a systematic review. J Med Virol. 2020;92(5): 479-90.

5. Chen P, Mao L, Nassis GP, Harmer P, Ainsworth BE, Li F. Coronavirus disease (COVID-19): the need to maintain regular physical activity while taking precautions. J Sport Health Sci. 2020;9(2):103-4.

6. Liang W, Guan W, Chen R, Wang W, Li J, Xu K, et al. Cancer patients in SARS-CoV-2 infection: a nationwide analysis in China. Lancet Oncol. 2020;21(3):335-7.

7. Conselho Federal de Nutricionistas. Nota Oficial: orientações para à população geral população e para os nutricionistas sobre o novo coronavírus. [citado 2020 Mar 10]. Disponível em: https:// www.cfn.org.br/index.php/destaques/19913/.

8. Campos LF, Barreto PA, Ceniccola GD, Gonçalves RC, Matos LBN, Zambelli CMSF, et al. Parecer BRASPEN/ AMIB para o enfrentamento do COVID-19 em pacientes hospitalizados. BRASPEN J. 2020;35 (Supl 1):3-5.

9. Sociedade Brasileira de Oncologia Clínica. Especial SBOC Coronavírus (COVID-19). [citado 2020 Mar 10]. Disponível em: https://sboc.org.br/images/ infograficooronav\%c3\%adrus_para_medicos_v5.pdf

10. Maia JE, Cruz LB, Gregianin LJ. Microbiological profile and nutritional quality of a regular diet compared to a neutropenic diet in a pediatric oncology unit. Pediatr Blood Cancer. 2018;65:e26828.

11. Morrell MBG, Baker R, Johnson A, Santizo R, Liu D, Moody K. Dietary intake and micronutrient deficiency in children with cancer. Pediatr Blood Cancer. 2019;66(10):e27895.

12. Horie LM, Barrére APN, Castro MG, Liviera AMB, Carvalho AMB, Pereira A, et al. Diretriz BRASPEN de terapia nutricional no paciente com câncer. BRASPEN J 2019;34 (Supl 1):2-32.

13. Sonbol MB, Jain T, Firwana B, Hilal T, Deleon T, Murad A, et al. Neutropenic diets to prevent cancer infections: updated systematic review and meta-analysis. BMJ Support Palliat Care. 2019;9(4):425-33.

14. Agência Nacional de Vigilância Sanitária. Nota técnica GVIMS/ GGTES/ANVISA nº 04/2020. Orientações para serviços de saúde: medidas de prevenção e controle que devem ser adotadas durante a assistência aos casos suspeitos ou confirmados de infecção pelo novo coronavírus (SARS-CoV-2). (atualizada em 21/03/2020). Brasília: ANVISA; 2020.

15. Brasil. Ministério da Saúde. Instituto Nacional de Câncer. Consenso nacional de nutrição oncológica para paciente pediátrico oncológico. Rio de Janeiro: Instituto Nacional de Câncer; 2014. 